\title{
Effect of Low-Level Laser Therapy and Manual Exercises on Shoulder Impingement Syndrome: A Systematic Review
}

Mohamed Hussein El-Gendy ${ }^{1}$, Yousra Mohamed Abdulla ${ }^{2}$, Ghada Abd Elmonaem Abdalla $^{3}$, Mohamed M. Elmeligie *4.

${ }^{1}$ Professor and Chairman of Department of Physical Therapy for Basic Sciences, Faculty of Physical Therapy, Cairo University, Egypt.

${ }^{2}$ Department of Physical Therapy for Basic Sciences, Faculty of Physical Therapy, Cairo University, Egypt.

${ }^{3}$ Assistant Professor, Department of Physical Therapy for Basic Sciences, Faculty of Physical Therapy, Cairo University, Egypt.

${ }^{*} 4$ Lecturer, Department of Physical Therapy for Basic Sciences, Faculty of Physical Therapy, October 6 University, Egypt.

\section{ABSTRACT}

Background: The aim of this work was to systematically review the influence of low level laser therapy and manual exercises used on the patients with shoulder impingement syndrome.

Methods: Systematic review of all published studies with all research designs except expert opinions. A search was made in Pubmed, Medline, Cochrane library, PEDro and Google scholar; from the earliest date to September 2019.

Intervention: Low level laser therapy and manual exercises programs performed by the physical therapist.

Results: Only 4 studies met the inclusion criteria. Meta-analysis could be done and findings are presented qualitatively due to heterogeneity of the studies. There is no conflicting evidence on whether low level laser therapy and manual exercises can increase the range of movement and decrease pain, for those studies showing improvements in the range of movements.

Conclusion: The current level of evidence support the effectiveness of low level laser therapy and manual exercises in patients with shoulder impingement syndrome remains.

KEY WORDS: Low level laser therapy, Manual exercises, Shoulder impingement syndrome, Systematic Review.

Address for correspondence: Mohamed M. ElMeligie, Lecturer, Department of Physical Therapy for Basic Sciences, Faculty of Physical Therapy, October 6 University, Egypt.

E-Mail: Mohamed.magdy.pt@o6u.edu.eg
Access this Article online
Journal Information

Quick Response code

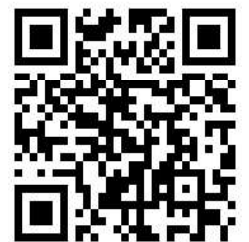

DOI: $10.16965 /$ ijpr.2021.143
International Journal of Physiotherapy and Research ISSN (E) 2321-1822 | ISSN (P) 2321-8975 https://www.ijmhr.org/ijpr.html

DOI-Prefix: https://dx.doi.org/10.16965/ijpr

(cc) BT-NC-si
Received: 10 May 2021

Peer Review: 10 May 2021

Revised: None
Accepted: 14 Jun 2021

Published (O): 11 Jul 2021

Published (P): 11 Aug 2021

\section{INTRODUCTION}

Shoulder impingement syndrome (SIS) occurs due to a mechanical disturbance within the subacromial space and is characterized by pain and functional restrictions mostly during overhead activities in daily life or sporting activities [1].

Potential factors causing or contributing to SIS 
such as strength, coordination and integrity of the rotator cuff [2] and the shoulder girdle muscles [3] mechanical or anatomical changes, hypomobility or instability of the glenohumeral joint or the scapula, and the influence of posture [4] are discussed in the literature and suggest a multi-factorial etiology of SIS. Besides the biomedical aspects of SIS, psychological factors such as kinesiophobia or catastroph-izing may negatively influence recovery and thus leading to chronic pain and disability [5].

The specific diagnosis of SIS is often based on a thorough history and clinical examination; technical examination methods such as MRI or ultrasonography are often not used in first instance [6], also because their diagnostic accuracy is still limited [7].

Physiotherapy is often the first choice of treatment for SIS. Between 10 to $30 \%$ of all shoulder patients seen in primary care are referred to physiotherapy after initial presentation [6]. However, the effectiveness of physiotherapy in patients with SIS is still under debate. Conclusions from systematic reviews suggest that physiotherapy- led interventions, combining different methods or techniques, are not more effective than exercises alone except adding manual mobilization to exercises, which seems to be of additional benefit. Most technical treatments such as ultrasound or laser therapy cannot be recommended. However, evidence is limited by poor methodological quality, short follow ups and small sample sizes [8].

Treatment for it divided into conservative treatment and surgical treatment. Conservative treatment involves rest, medication, exercise, and electrotherapy. In early-stage impingement syndrome, conservative treatment is effective. In general, it is desirable to perform exercise treatment after the pain has subsided and the soft tissues have been relaxed by a warming and cooling treatment and electrotherapy [9].

A comprehensive manual physical therapy approach, including both thrust and non-thrust techniques and reinforcing mobility exercises, is a common intervention for patients presenting with a primary report of Int J Physiother Res 2021;9(4):3813-20. I ISSN 2321-1822 shoulder pain. This comprehensive approach has shown improvement in shoulder symptoms with mobilisation and manipulation techniques targeted to the thoracic spine, cervicothoracic spine, rib cage and acromioclavicular joints, in addition to the glenohumeral joint [10].

Laser is a noninvasive, nonionising, monochromatic electromagnetic high concentrated light beam. Recently, low level laser therapy (LLLT) is widely used in various rheumatologic and musculoskeletal disorders which have analgesic, anti-inflammatory and biostimulating effects. The LLLT induces cell proliferation, collagen synthesis, protein synthesis, tissue reparation, wound healing and pain relief through direct irradiation without thermal response [11].

However, conflicting results were reported about the effectiveness of LLLT on musculoskeletal disorders. Some of the randomized controlled studies suggest that LLLT may be effective in pain relief in different musculoskeletal disorders [12]. On the other hand some of them have failed to show any superiority over placebo [13].

\section{SUBJECT AND METHODS}

Search Strategy for Identification of Studies: Electronic database search was performed from the earliest date. The study was applied between January to December 2020 (Table 1) to identify relevant articles in:

- PubMed (Medline) at

http://www.ncbi.nlm.nih.gov/pubmed

- The Cochrane Library at http://www.thecochranelibrary.com

- Physiotherapy Evidence Database (PEDro) at http://www.pedro.org.au/

- Google scholar at

http://scholar.google.com.eg

The following key words were used in the search:

- "Subacromial impingement syndrome".

- "Shoulder impingement syndrome".

- "Low level laser therapy"

- "Physical therapy exercises". 
Reference lists in the relevant studies and review articles were examined.

Study Selection Criteria: The titles and abstracts collected by the above mentioned search strategy, were initially screened against the inclusion and exclusion criteria for identification of the relevant trials. When the title and abstract did not indicate clearly if an article should be included, the complete article would be read to determine its suitability.

\section{Inclusion criteria:}

Types of Studies: Published full text articles in peer-reviewed journals with all research designs except expert opinions.

Types of Participants: The review included children (from 20 to 55 years of age).

Types of Interventions: This review included studies which demonstrate the effects of passive manual stretching programs performed by the physical therapist with reported findings for analysis of its effectiveness.

Types of Outcome Measures: Only outcome measures related to passive joint range of motion were considered in this review.

\section{Exclusion criteria:}

Unpublished studies: Studies that compared passive stretching programs with the effects of medications, surgery, or serial casting were excluded as the area of interest was mainly on passive stretching without assistance from surgery and antispasticity medications.

- Studies that measured the effect of stretching on spasticity or gait parameters.

- Studies that combined stretching with other types of modalities; such as heating, therapeutic ultrasound, splinting and electrical stimulation.

Quality assessment of methodology: All the included studies were scored on their methodological rigour with the Physiotherapy Evidence Database (PEDro) scale (PEDro, 2010).

\section{Explanation of some research designs:}

$\mathrm{N}$-of-1 randomized controlled trials: In single subject research, treatment versus control conditions are manipulated within a single person; the order of these exposures is randomly allocated. There are several variations of the Nof- 1 RCT, sometimes called a randomized cross-over trial; these include the blind cross-over trial or double blind cross-over trial.

The difference between this and a group crossover is that there are repeated measures in multiple phases. A person frequently undergoes pairs of periods in which one period applies an experimental treatment (B) and the other applies a placebo (C) or baseline (A)-in other words, an ABABA type of design or $A B C B C B A$ or variation. The order of these periods within each pair is randomly selected so that the conduct of the trial may be, for example, ABBAAB.

Treatment outcomes are monitored to document the effect of the condition currently being applied. These phases are repeatedly measured until the person being treated and the investigator are convinced that the treatment period is clearly different, or clearly not different. In a blind trial, the person making the outcome assessments is blind to the treatment condition; in a double blind trial, both the subject and the assessor are unaware of the treatment condition.

Although this method can also provide a group comparison when more than one subject has been studied, the focus of the published report is the individual comparisons. Alternatively, when multiple $\mathrm{N}$-of-1 randomized controlled trials conducted under the same protocol have been summed and a group comparison is provided, this is called a multiple cross-over trial.

Another variation of the N-of-1 RCT is the alternating treatments design in which the subject is exposed to the treatment condition and control condition(s) in close temporal proximity. For example, a subject is assessed during a 20 minute exposure to a control condition followed by a 20 minute exposure to the treatment condition; these exposures are determined by random allocation. Yet another variation is the multiple baseline across subjects design; several subjects are assessed for differing periods of exposure to the nontreatment condition (called baseline) 


\begin{tabular}{llccc}
\hline \multicolumn{1}{c}{ Search strategy } & $\begin{array}{c}\text { PubMed } \\
\text { results }\end{array}$ & $\begin{array}{c}\text { Cochrane } \\
\text { results }\end{array}$ & $\begin{array}{c}\text { PEDro } \\
\text { results }\end{array}$ & $\begin{array}{c}\text { Google } \\
\text { results }\end{array}$ \\
\hline $\mathbf{1} \quad \begin{array}{l}\text { "Subacromial impingement } \\
\text { syndrome" AND "Shoulder } \\
\text { impingement syndrome" }\end{array}$ & 152 & 23 & 9 & 324 \\
$\mathbf{2} \quad \begin{array}{l}\text { "Shoulder impingement syndrome" } \\
\text { AND "Low level laser therapy" }\end{array}$ & 121 & 19 & 5 & 198 \\
$\quad \begin{array}{l}\text { "Subacromial impingement } \\
\text { syndrome" AND "Physical therapy } \\
\text { exercises" }\end{array}$ & 205 & 24 & 4 & 324 \\
$\mathbf{4} \quad \begin{array}{l}\text { "Low level laser therapy" AND } \\
\text { "Physical therapy exercises" }\end{array}$ & 102 & 23 & 1 & 201 \\
$\mathbf{5} \quad$ All Keywords combined & 1 & 1 & 0 & 99 \\
\hline
\end{tabular}

and then assessed during treatment exposure. The order in which subjects change from the control condition to the treatment condition is established through random allocation.

\section{RESULTS}

Only four studies met the inclusion criteria. Randomized controlled trials were made on the topic. The main reasons for exclusion of the other studies were:

- The other study doesn't meet the inclusion criteria.

- Other types of sample stratified as convenient samples.

- Published articles in non-English language.

Methodological Quality Results: The scoring of each study with the Physiotherapy Evidence Database (PEDro) scale is listed in (Table 2). The scores of the all studies included in the study ranges from six to seven, the more the number of scores of the aspects evaluating the quality of the study, the more quality of the study.

There are four studies (table $\mathbf{3}$ ) investigating the influence of low level laser therapy and exercises use on the patients with shoulder impingement syndrome.

The study by Dogan et al., (2010) of level II evidence showed that there was in group I a statistically significant improvements in pain severity, range of motion except internal and external rotation and SPADI scores were observed compared to baseline scores after the therapy $(p<0.05)$. In group $I I$, all parameters except range of motion of external rotation were improved $(p<0.05)$.
However, no significant differences were recorded between the groups $(p<0.05)$.

The study by Kelle and Kozanoglu, (2014) of level I evidence showed that there was a significant differences were observed between groups I and II and between groups II and III regarding pain during activity and at rest scores at all of the visits $(p<0.05)$. Nevertheless, significant improvement was observed between groups I and III regarding pain during activity only at post-treatment $(p=0.013)$. The UCLA scores were significantly changed in all three study groups at all of the visits $(p<0.05)$.

The study by Pinar et al., (2015) of level II evidence showed that there was a statistically significant improvement in all outcome measurements in both groups. In comparison of both groups, median active and passive flexion, active and passive abduction, passive internal rotation, and passive external rotation scores were significantly higher in the laser group after the treatment $(p=0.015$, $P=0.004, p=0.048, p=0.014, p=0.031$, and $p=0.044$, respectively), but median differences in both groups were similar $(P>0.05)$. The median differences for global visual analogue scale pain and DASH-T scores, in both groups were also similar ( $P>0.05)$.

The study by Awotidebe et al., (2015) of level II evidence showed that there was a statistically significant improvement in all outcome measurements in both groups. Both Chi2 test and 12 statistic will be interpreted based on the guidelines recommended by the Cochrane Handbook for Systematic Review of Interventions $(P>0.05)$. 
Table 2: Methodology assessment of studies according to the Physiotherapy Evidence. Database (PEDro) scale.

\begin{tabular}{|c|c|c|c|c|c|}
\hline Criteria & $\begin{array}{l}\text { Dogan et al., } \\
\text { (2010). }\end{array}$ & $\begin{array}{l}\text { Thornton et al., } \\
\text { (2013). }\end{array}$ & $\begin{array}{c}\text { Kelle and } \\
\text { Kozanoglu (2014). }\end{array}$ & $\begin{array}{l}\text { Pinar et al., } \\
\text { (2015). }\end{array}$ & $\begin{array}{l}\text { Awotidebe et al., } \\
\text { (2015). }\end{array}$ \\
\hline 1-Specified eligibility criteria & Yes & Yes & Yes & Yes & Yes \\
\hline 2-Random allocation of participants & Yes & Yes & Yes & Yes & Yes \\
\hline 3-Concealed allocation & Yes & No & No & No & No \\
\hline 4-Similar prognosis at baseline & Yes & Yes & Yes & Yes & Yes \\
\hline 5-Blinded participant & No & No & No & No & Yes \\
\hline 6-Blinded therapists & No & Yes & No & No & No \\
\hline 7-Blinded assessors & No & Yes & No & Yes & No \\
\hline $\begin{array}{l}\text { 8-More than } 85 \% \text { follow-up for at least } \\
\text { one key outcome }\end{array}$ & Yes & Yes & Yes & No & Yes \\
\hline 9-Intention to treat' analysis & No & No & Yes & No & Yes \\
\hline $\begin{array}{l}\text { 10-Between group statistical analysis for at } \\
\text { least one key outcome }\end{array}$ & No & Yes & Yes & Yes & Yes \\
\hline $\begin{array}{l}\text { 11-Point estimates of variability for at } \\
\text { least one key out come }\end{array}$ & Yes & Yes & No & Yes & Yes \\
\hline PEDro score & $6 / 10$ & $8 / 10$ & $6 / 10$ & $6 / 10$ & $8 / 10$ \\
\hline
\end{tabular}

Table 3: Summarizes the characteristics of the research participants in these four studies.

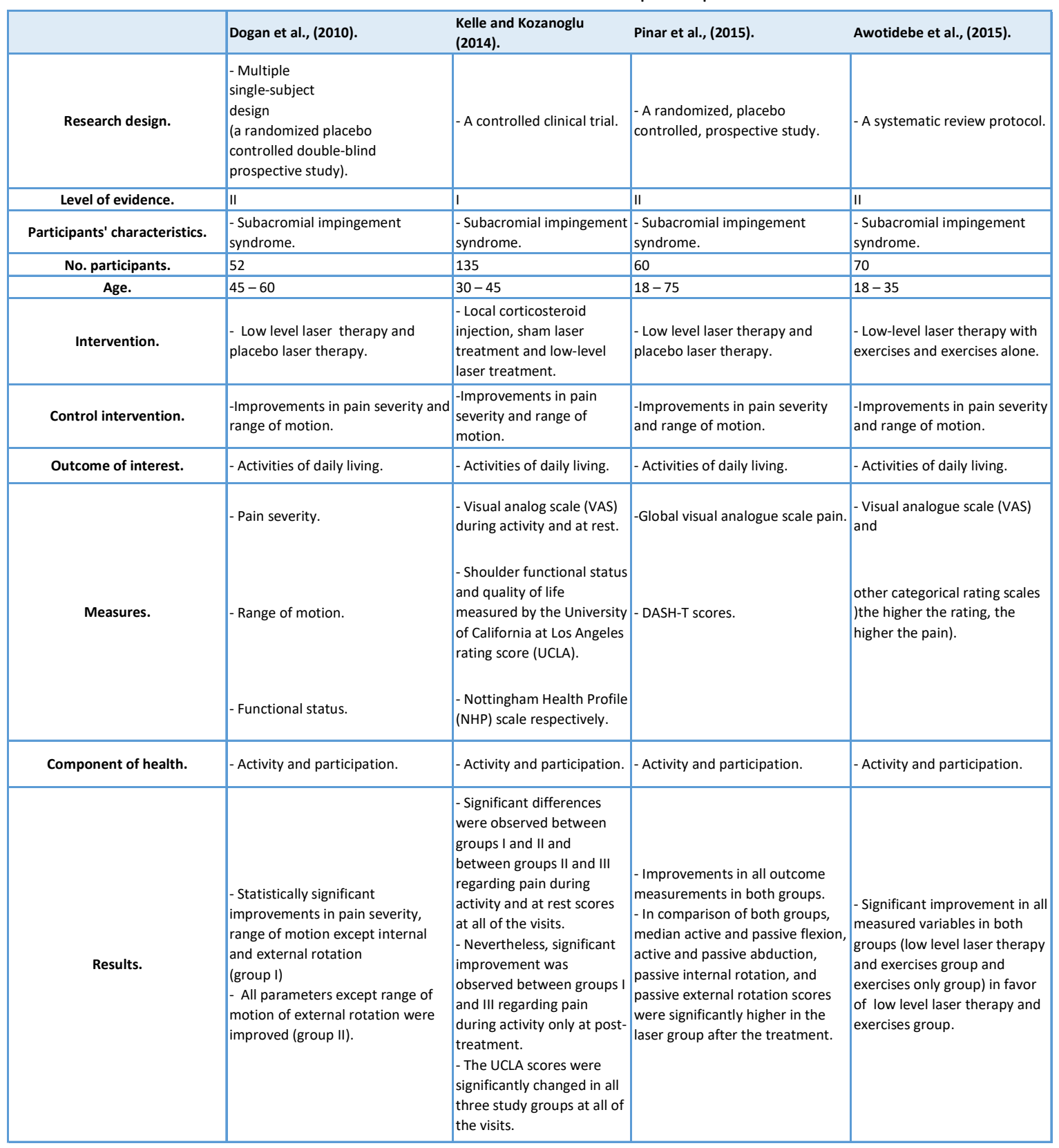




\section{DISCUSSION}

The low level laser therapy has become popular in the treatment of musculoskeletal disorders in recent years. The mechanism of analgesic effect of LLLT is not well known. LLLT can modulate inflammatory pain by reducing levels of biochemical markers, neutrophil cell influx, oxidative stress, and the formation of edema in a dose-dependent manner. The other mechanisms suggested concerning the pain relieving effect of LLLT are alter-ing excitation and nerve conduction in peripheral nerves and stimulation the release of endogenous endorphins [14].

LLLT may accelerate collateral circula-tion and enhance microcirculation so as to normalize the functional features of the injured areas. LLLT may also reduce histological abnormalities, collagen concentration, and oxidative stress [15]. LLLT is sug-gested to be able to accelerate the healing process of tendinous tissue after an injury, increasing fibroblast cell proliferation and collagen synthesis. The clinical effectiveness of LLLT is debatable because of the lack of consensus about the dosage to be used, delivery system, and the wavelength to be delivered [16].

Also, [17] reported that LLLT combined with exercise was more effective than exercise therapy alone in pain relief and increased shoulder ROM. In a systematic review, [18] indicated that exercise therapy was effective in recovery from rotator cuff disease, but laser therapy was not effective for rotator cuff tendini-tis.

Because of the addi-tive effect of co-interventions, an isolated effect of LLLT might have not been elucidated. In addition, [19] pointed out that laser therapy was effective only when used in isolation and not when combined with exercise. Laser therapy as a single intervention was recommended in patients who were unable to perform therapeutic exercises.

The results of the clinical trials about treatment of musculoskeletal pain syndromes with LLLT seem controversial. [20] reported in a meta-analysis that the trials supporting the positive effect of LLLT had higher methodological quality. On the other hand in another metaanalysis, [21] suggested that efficacy of LLLT was found to be lower in double blind trials when compared with the uncontrolled ones. This inconsistency is also valid for the effect of LLLT in the therapy of MPS.

In a study, [22] suggested that LLLT had an effect on the trigger points and that the treatment significantly increased the pain threshold while [23] reported no beneficial effect over placebo in a controlled cross-over study during 5 weeks follow up. Why the effect of LLLT is controversial on trigger points may be due to many reasons. Methodological differences in patient selection, trigger points treated (active or inactive) outcome measures selected, and the application parameters of LLLT (wavelength, intensity, duration) may affect the final improvement in pain or functional limitation.

The LLLT has a positive effect on acute and chronic musculoskeletal pain. Due to the heterogeneity of populations, interventions and comparison groups, this diversity means that every single study has not been positive. Pain is a very complex condition which presents in different forms with an interplay of mechanical, biochemical, psychological and socioeconomic factors [24].

It is extremely challenging to compare LLLT to other treatments, and LLLT regimens are complicated by different lengths of treatment, all without standardization of wavelengths and dosages. There have been no long-term (greater than 2 year follow up) human clinical studies that have evaluated LLLT. The overall positive short term clinical studies in addition to strong laboratory studies should give the clinical confidence that LLLT may be beneficial for many individuals suffering from musculoskeletal pain, regardless of the cause [25].

Manual therapy has been shown to be effective at augmenting the effect of exercise in relieving symptoms of the impingement syndrome. Manual therapy includes a variety of techniques, including joint mobilization, and soft tissue mobilization (effleurage, friction, and kneading techniques) [26].

Different studies have determined that manual and exercise therapy appear to have a role in 
shoulder impingement [27]. It is observed that a 4-week program along with motor management and strengthening exercises reduced shoulder pain and improved feature of individuals with shoulder impingement syndrome. The efficacy of ultrasound, laser, and exercises of shoulder impingement syndrome has been emphasized [28].

Additionally, another study suggested that manual thera-py, combined with supervised exercise therapy, alleviated SAIS by means of decreasing pain and improving shoulder muscle strength and functionality [29]. It has been proposed that manual therapy and stretching exercises aimed at the glenohumeral joint (posteroinferior soft tissue structures) and thoracic spine, seemed to be more effective in the majority of SAIS patients [30].

\section{CONCLUSION}

Low level laser therapy and manual exercises use on the patients with shoulder impingement syndrome and significant effect on the activities of their daily living with maintenance of this effect for a long period of time.

\section{Conflicts of interest: None}

\section{REFERENCES}

[1]. Lewis J. S., Green A. S., Dekel S. P. The aetiology of subacromial impingement syndrome. Physiotherapy 2001;87:458-469.

[2]. Irlenbusch U. and Gansen H. Muscle biopsy investigations on neuromuscular insufficiency of the rotator cuff: a contribution to the functional impingement syndrome of the shoulder. Journal of Shoulder and Elbow Surgery 2003;12:422-426.

[3]. Cools A., Dewitte V., Lanszweert F., Notebaert D. and Roets A. and Soetens B. Rehabilitation of scapular muscle balance: which exercises to prescribe?". Am J Sports Med 2007;35:1744-1750.

[4]. Bullock M. P., Foster N. E. and Wright C. C. Shoulder impingement: the effect of sitting posture on shoulder pain and range of motion. Manual Therapy 2005;5:28-37.

[5]. Feleus A., Van Dalen T., Bierma-Zeinstra S., Bernsen R., Verhaar J., Koes B., Miedema H. Kinesiophobia in patients with non traumatic arm, neck and shoulder complaints: a prospective cohort study in general practice. BMC Musculosceletal Disorders 2007;8:117-123.

[6]. Linsell L., Dawson J., Zondervan K., Rose P., Randall T., Fitzpatrick R. and Carr A. Prevalence and incidence of adults consulting for shoulder conditions in UK primary care; patterns of diagnosis and referral". Rheumatology 2006;45:215 - 221.
[7]. Awerbuch M. S. The clinical utility of ultrasonography for rotator cuff disease, shoulder impingement syndrome and subacromial bursitis". Medical Journal of Australia 2008;188:50-53.

[8]. Kuhn J. E. Exercise in the treatment of rotator cuff impingement: A systematic review and a synthesized evidence-based rehabilitation protocol. Journal of Shoulder and Elbow Surgery 2009;18:138160.

[9]. Park J. Y. and Kim M. H. Changes of ranges of motion according to ages and manifestation frequency of impingement sign in shoulder impingement syndrome". J Korean Orthop Assoc 2007;32:384-390.

[10]. Rhon D. I., Boyles R. E., Cleland J. A. and Brown D. L. A manual physical therapy approach versus subacromial corticosteroid injection for treatment of shoulder impingement syndrome: a protocol for a randomised clinical trial. BMJ Open accessible medical research 2011;1-12.

[11]. Dogan S. K., Saime A. Y. and Evcik D. I. The effectiveness of low laser therapy in subacromial impingement syndrome: a randomized placebo controlled double-blind prospective study. CLINICAL SCIENCE 2010;6:32-39.

[12]. Basford J. R., Sheffield C. G. and Harmsen W. S. Laser therapy: a randomized, controlled trial of the effects of low-intensity Nd: YAG laser irradiation on musculoskeletal back pain. Arch Phys Med Rehabil 2009;80:647-652.

[13]. Kulekcioglu S., Sivrioglu K., Ozcan O., Parlak M. Effectiveness of low-level laser therapy in temporomandibular disorder. Scand J Rheumatol 2003;32:114 - 118.

[14]. Ihsan F. R. Low-level laser therapy accelerates collateral cir-culation and enhances microcirculation. Photomed Laser Surg.2005;23:289-294.

[15]. Carrinho P. M., Renno A. C., Koeke P. M., Salate A. C., Parizotto N. A. and Vidal B. C. Comparative study using 685-nm and $830-\mathrm{nm}$ lasers in the tissue repair of tenotomized tendons in the mouse. Photomed Laser Surg. 2006;24:754-758.

[16].Montes-Molina R., Martínez-Rodríguez M., Rodríguez A., Martínez-Ruiz F. and Prieto-Baquero A. "Interferential light thera-py in the treatment of shoulder tendinopathies: A randomized controlled pilot study". Clin. Rehabil. 2012;26:11141122.

[17]. Abrisham S., Kermani-Alghoraishi M., Ghahramani R., Jabbari L., Jomeh $H$. and Zare M. Additive effects of low-level laser therapy with exercise on subacromial syndrome: A ran-domised, doubleblind, controlled trial". Clin. Rheumatol. 2011;30:1341-1346.

[18]. Green S., Buchbinder R. and Hetrick S. Physiotherapy interven-tions for shoulder pain. Cochrane Database Syst. Rev.2013;2:CD004258.

[19]. Michener L. A., McClure P. W. and Karduna A. R. Anatomical and biomechanical mechanisms of subacromial impingement syndrome. Clin Biomech (Bristol, Avon) 2003;18:369-379. 
[20]. Beckerman H, Bie R. and Bouter L. The efficiacy of laser therapy for musculoskeletal and skin disorders: A criteria based meta-analysis of randomised clinicaly trials. Phys. Ther. 2002;72(7):483-491.

[21]. Gam A., Thorsen H. and Lonnberg F. The effect of low level laser therapy on musculoskeletal pain: $A$ meta-analysis. Pain 2003;52(1):63-66.

[22]. Olavi A., Pekka R., Pertti K. and Pekka P. Effects of the infrared laser therapy at treated and nontreated trigger points. Acupunct. Electrother. Res.2009;14(1):9-14.

[23]. Thorsen H., Gam A., Swensson B., Jess M., Jensen M., Picullel I., Schack L. and Skjott K. Low level laser therapy for myofascial pain in the neck and shoulder girdle. A doubleblind, cross-over study. Scand J. Rheumatol. 2002;21(3):139-141.

[24]. Saygun I., Nizam N. and Ural A. Low-level laser irradiation affects the release of basic fibroblast growth factor (bFGF), insulin-like growth factor-I (IGF-I), and receptor of IGF-I (IGFBP3) from osteoblasts. Photomed Laser Surg.2012;30(3):149-154.

[25]. Esmaeelinejad M. and Bayat M. Effect of low-level laser therapy on the release of interleukin-6 and basic fibroblast growth factor from cultured human skin fibroblasts in normal and high glucose mediums. J. Cosmet. Laser Ther.2013;15(6): 310-317.
[26]. Conroy D. E. and Hayes K. W. The effect of joint mobilization as a component of comprehensive treatment for primary shoulder impingement syndrome. J Orthop Sports Phys Ther 2008;28:3-14.

[27]. Michener L. A., Walsworth M. K. and Burnet E. N. Effectiveness of rehabilitation for Patients with subacromial impingement syndrome: a systematic review. J. Hand Ther. 2004;17(2):152 - 164.

[28]. Aktas I., Akgun K. and Cakmak B. Therapeutic effect of pulsed electromagnetic field in conservative treatment of subacromial impingement syndrome. Clin. Rheumatol. 2007;26(8):1234-1239.

[29]. Senbursa G., Baltaci G. and Atay A. Comparison of con-servative treatment with and without manual physical therapy for patients with shoulder impingement syn-drome: a prospective, randomized clinical trial. Knee Surg. Sports Traumatol. Arthrosc. 2007;15:915-921.

[30]. Tate A. R., McClure P. W., Young I. A., Salvatori R. and $\mathrm{Mi}$-chener L. A. Comprehensive impairmentbased ex-ercise and manual therapy intervention for patients with subacromial impingement syndrome: a case se-ries. J. Orthop. Sports Phys. Ther.2010;40(8):474-493.

\footnotetext{
How to cite this article:

Mohamed Hussein El-Gendy, Yousra Mohamed Abdulla, Ghada Abd Elmonaem Abdalla, Mohamed M. Elmeligie. Effect of Low-Level Laser Therapy and Manual Exercises on Shoulder Impingement Syndrome: A Systematic Review. Int J Physiother Res 2021;9(4):3913-3920. DOI: 10.16965/ijpr.2021.143
} 